\title{
Comparison between Duobinary Filter and Delay-And-Add Technique of Duobinary Transmission in Fiber Optic Communication System
}

\author{
Tawsif Hyder ${ }^{1}$ \\ ${ }^{I}$ (Department of Electrical and Electronic Engineering/Ahsanullah University of Science and Technology, \\ Bangladesh)
}

\begin{abstract}
Binary code is a two level of bit sequence of 1 and 0 . On the other side, Duobinary (DB) is a threelevel code of 1, 0 and -1 levels. Using duobinary transmission instead of binary transmission, it can improve the system performance, bandwidth efficiency of output signal, average power of signal, noise and distortion ratio of output wave shapes. Duobinary encoding and decoding are used in a significant bandwidth reduction of MAC signals transmitted by high and medium power TV satellites and also used in fiber optic communication system. This paperis based on the the observationof generation performance and transmission of duobinary coding and simulation of a single channel (1550-nm) 90-km OC-192 link. In duobinary transmission, the duobinary encoding can be implemented either with a combination of a delay-and-add operation or with a duobinary filter. Duobinary filter reduces bandwidth, wavelength, noise and distortion and improves the eye diagram of the output signals than the delay-and-add circuit in duobinary transmission of optical communication system. Also in this paper circuit design, simulation and fabrication of a duobinary encoder and decoder circuit for data communication system are presented.
\end{abstract}

Keywords: Duobinary transmission, Duobinary filter, Delay-and-add circuit, Optsim, Eye diagram.

\section{Introduction}

Ideas from communication theory developed originally for microwaves and electrical signals are often applied signals. Duobinary transmission is one such idea that was development in the early 1960's in the context of electrical signals and was later applied to optical signals in the late 1990's.

As the demand for faster communication increases there has been a natural evolution towards a better usage of channel bandwidth. In the radio and microwave domains, radios, televisions and especially cellular phones have led to efficient usage of the scarce and precious channel spectrum, through the usage of bandwidth efficient modulation techniques; single-side band modulation and time division multiplexing. In comparison, Optical communications in fiber has a large usable bandwidth. In optical fiber communications, binary NRZ modulation is still currently the most popular modulation format in optical fibers due to its simplicity of implementation. One way of achieving a more efficient use of the channel bandwidth was through innovative coding schemes called partial response signaling (PRS). PRS is a method of encoding or decoding a data stream in order to decrease the amount of error from inter symbol interference (ISI). Duobinary format is a common form of partial response signaling. A binary data stream can be encoded into a duobinary signal simply by adding the binary data stream to a one bit delayed version of itself. The result is a three-level signal.

Lender was one of the first to publish on duobinary signaling [37-38]. He pointed out that the advantage of using duobinary signaling in electrical lines is that a bit rate of $2 \mathrm{~B}$ can be sustained in a channel of single-sided bandwidth B with a reduced amount of ISI and without the need for ideal low-pass rectangular base band filters as would be necessary to achieve the same performance using binary format.

Up until the 1990's fiber optic communication had plenty of bandwidth. Just as had happened in the microwave spectrum in the 1960's bit rates and number of channels in optical fibers started to increase and fill the $3 \mathrm{THz}$ bandwidth of erbium-doped fiber amplifiers (EDFA). Very quickly, the bandwidth of the entire amplifier was used by densely-packed wavelength-division multiplexed (WDM) binary channels. The bandwidth of EDFA was to increase the spectral efficiency (the bit rate divided by the used bandwidth) by using a different modulation format. Duobinary format is the natural one improving the bandwidth efficiency since it has a compressed spectrum and the receiver does not have a local-oscillator. In the 1997 Optical Fiber Conference, the feasibility of over $1 \mathrm{bit} / \mathrm{s} \mathrm{Hz}$ high spectral efficiency WDM with optical duobinary coding and polarization interleave multiplexing was considered [39].

Besides these a more efficient use of available optical bandwidth, the recent interest in duobinary signaling in optical fibers grew from its low stimulated Brillouin scattering (SBS) threshold and its narrow spectrum. The spectral width of duobinary signals is about half of that of binary signals and since a narrower spectrum implies a smaller dispersion power penalties. Duobinary signals help alleviate the problem of ever 
increasing modulation rates. Stimulated Brillouin backscattering (SBS) presents an upper limit to the launch power of an NRZ signal. This upper limit is called the SBS threshold. Duobinary Signals have higher SBS thresholds than binary signals since binary signals have a large spectral component at the carrier frequency, not present in the duobinary power spectral density (PSD), which efficiently scatters from acoustic waves in the fiber at high launch powers.

The rediscovery of duobinary transmission in optical fibers has prompted a deluge of research to use this transmission format to increase capacity of fiber while still offering reasonable hardware (receiver and transmitter) realizations. One of the first duobinary experiments (in 1994) was the propagation of a three-level optical duobinary signal over an unrepeated 100km span of silica core fiber [8]. One year later (1995), a simple optical modulation scheme yielding and AM-PSK duobinary optical signal using a lithium niobate Mach-Zender modulator driven by an electrical three-level duobinary signal was proposed [40]. This scheme proved to be advantageous over the previous three-level optical level scheme since in yielded a two-level intensity signal in the fiber which allowed for direct detection and a better back-to- back sensitivity. In the same year a $210 \mathrm{~km}$ repeater less $10 \mathrm{~Gb} / \mathrm{s}$ transmission experiment through no dispersion-shifted fiber $(17 \mathrm{ps} / \mathrm{nm} / \mathrm{km})$ with a measured bit error ratio lower the $10^{-12}$ was performed [41]. To summarize, the four attractive features of duobinary modulation in optical fiber transmission are: (1) it has a greater spectral efficiency than binary format due to its narrower bandwidth (2)allows tighter packing of wavelength division multiplexed channels, (3) it suffers less from stimulated Brillouin Backscattering, the major limiting factor in repeater less transmission, and (4) is easy to implement since the transmitter only requires modest changes from and externally modulated binary transmission and since the receiver is a direct detection receiver, the same as for binary format.

\subsection{Duobinary Transmission Based Fiber Optic Communication System}

As like as other communication system, the fiber optic communication have three parts: transmitter, channel and receiver.Block diagram of a duobinary filter based and delay-and-add technique based duobinary fiber optic communication systems are shown in figure 1.1 and 1.2 respectively. In this case message means an electrical or other form of signal. This message is transmitted by a transmitter and passed through the channel. It is detected and converted to the required form by the receiver. The electrical section of the duobinary transmitter consists of a data generator, a duobinary encoder and a low-pass filter.

Transmitting Side

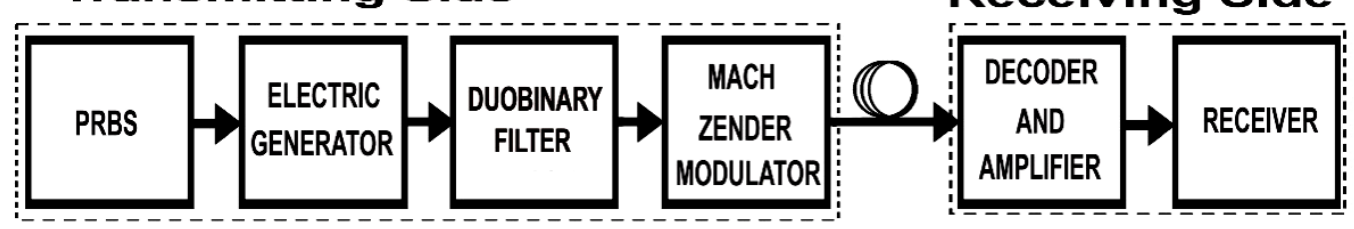

Fiber Channel

Figure 1.1 General block diagram of duobinary filter in duobinary transmission system

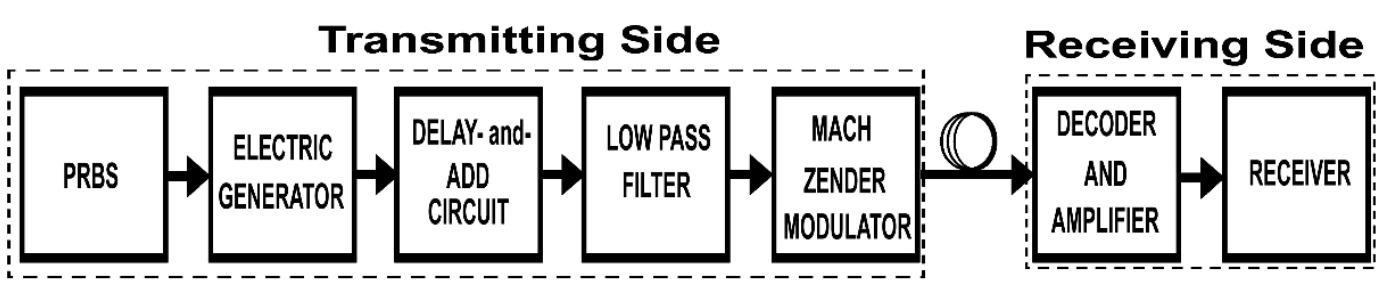

Fiber Channel

Figure 1.2 General block diagram of Delay-and-Add operation in duobinary transmission System

For transmission experiments, the data generator is often a pseudo-random bit sequence which has properties of random data. To the transmitting side, the main function of the precoder is to produce the errorless duobinary signal. The encoder encoded the duobinary signal either with delay-and-add circuit or with duobinary filter. In fiber optic communication system laser or light emitting diode are used as an optical oscillator or a carrier signal. The purpose of low pass Bessel Thompson filter is to generate the three levels duobinary electrical signal. Then the signal is modulated by Mach-Zender Modulator (MZM). They generate signal frequency with sufficient power to travel long distance. The amount of power radiation is proportional to the output current of the modulator. This modulated signal is transmitted through the proper channel means fiber 
optic cable. It may be guided or non-guided. This signal is demodulated to the receiving end, decoded and filtered. Receiver detects, amplifies and converts the signal to proper form.

\subsubsection{Pseudo Random Binary Sequence (PRBS)}

Pseudo-random data generator has three properties that reflect the data's randomness [42]. Firstly, the number of marks and spaces (or zeros and ones) in a sequence differ by at most 1 . Secondly, the probability of a continuous string of marks or spaces is inversely proportional to the length of the string. This means that among the number of runs of marks of spaces in the pseudorandom binary sequence (PRBS), one-half the funs of each kind are of length one, one-fourth are length two, one-eighth are length three, and so on. Finally, the autocorrelation of the PRBS is approximately zero everywhere except at the origin. The generation of a PRBS is implemented by using a shift register with feedback has three properties that reflect the data's randomness [42].

\subsubsection{Optical Duobinary Signal from Binary Bit}

Duobinary data can be achieved from binary data by adding the binary bits and shifting the bit stream to itself. For example, the binary bit sequence is 10110010.The shifted first bit will be 0(zero).The transfer function of duobinary encoder is $\mathrm{H}(\mathrm{z})=1+\mathrm{z}^{-1}$. To the decoder side

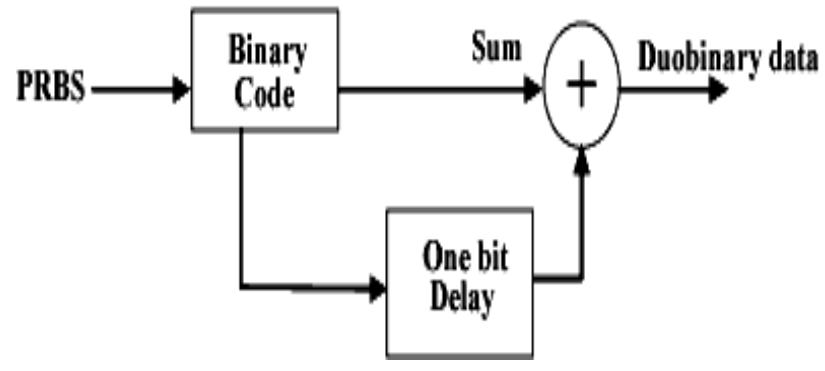

Figure 1.3 Block diagram of converting duobinary code from binary

The inverse transfer function will be $\mathrm{H}(\mathrm{z}) \mathrm{G}(\mathrm{z})=1$ or $\mathrm{G}(\mathrm{z})=1 /\left(1+\mathrm{z}^{-1}\right)$ which is described in figure 3.3. One problem in duobinary encoding is that the decoder output depends on previous bits which are solved by using precoding technique. The transfer function of precoding is $\mathrm{P}(\mathrm{z})=1 /\left(1-\mathrm{z}^{-1}\right)[1]$.

\begin{tabular}{|c|c|c|c|c|c|c|c|c|c|c|}
\hline ice: & 1 & & 1 & 1 & 0 & 0 & & & 0 & $\mathrm{X}$ \\
\hline elayed Binary & (0) & 1 & 0 & 1 & 1 & 0 & & 0 & 1 & P \\
\hline uob & 1 & 1 & 2 & 1 & 0 & 1 & & 1 & $\mathrm{X}$ & \\
\hline Duobinary in or & & 0 & 0 & 0 & & & -1 & 0 & & \\
\hline
\end{tabular}

Table 1.1 Example of converting duobinary code from binary

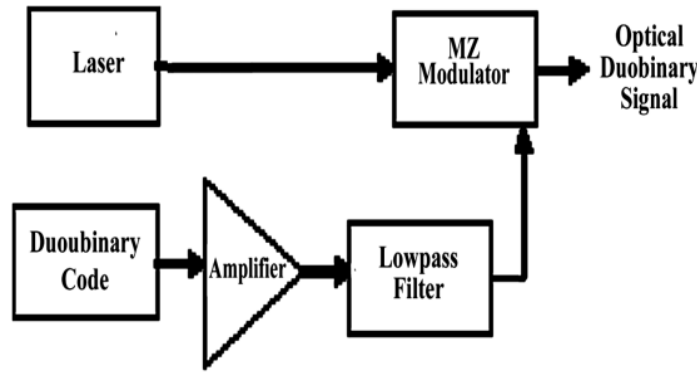

Figure 1.4 Block diagram of generating optical duobinary signal

From table 1.1 the duobinary code is a three level coding technique which are indicated $-1,0,1$ instead of having levels $0,1,2$.The upper and lower levels are differing $\pi$ phase from each other. Mach zender uses the technique of amplitude modulated phase shift keying AM-PSK duobinary modulation. The optical duobinary signal can be achieved after modulation with the combination of duobinary-coded signal and laser by MZM [1].

\subsubsection{Precoder, Duobinary Encoder and Decoder}

Optical duobinary coding, is an effective method for high-speed optical transmission system to improve the spectral efficiency and to increase the dispersion tolerance. Duobinary signals require less 
bandwidth than NRZ and permits the detection of some errors without the addition of error-checking bits. In the decoding of the duobinary signal, it is noticed that the decoding of the present bit value depends on the correct bit and decoding of the previous bit value.

This is a problem as decoding that errors tend to propagate [6].In that case the precoder at the transmitter is required in order to allow easy data recovery at the receiver. Without a precoder the binary data signal can be recovered (using the inverse functionality of the encoder) but the current data depends on the current bit and on the previous bit. Such a recursive receiver is sensitive to detect error propagation and is therefore not practical to ensure hundred percent error corrections [2].

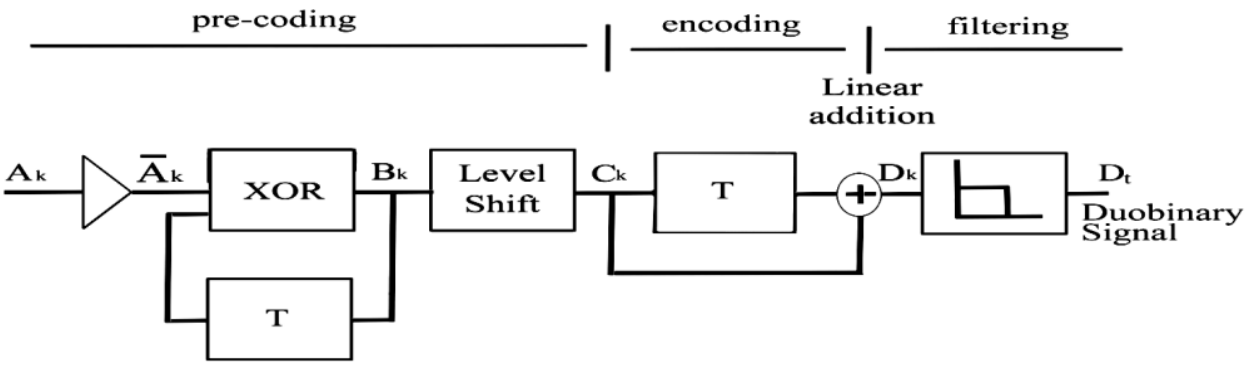

Figure 1.5 Basic structure of a duobinary encoder

Up to now, almost all optical duobinary transmission experiments were performed without a precoder. Due to the properties of the commonly used pseudo random bit sequences (PRBS). The precoder output bit stream is identical to its time delayed input bit stream. Therefore, the functionality of the precoder can be omitted for laboratory use but for transmitting actual data traffic with duobinary coding without a precoder is not possible.

\subsubsection{Mach Zender Modulator (MZM)}

The Duobinary electrical signal drives the Mach Zender Modulator (MZM) to produce optical Duobinary signal. A Mach-Zender modulator (MZM) is capable of modulating both the amplitude and phase. A duobinary Mach-Zender (MZ) Modulator is shown in figure 1.6. A continuous wave or pulsed light wave generated by a laser diode is modulated by an external MZ modulator. The two arms of the MZ modulator are driven by two electrical signals in push-pull fashion. The laser diode and the Mach-Zender (MZ) modulator make up the optical section of the duobinary transmitter means optical duobinary signal. The Mach-Zender

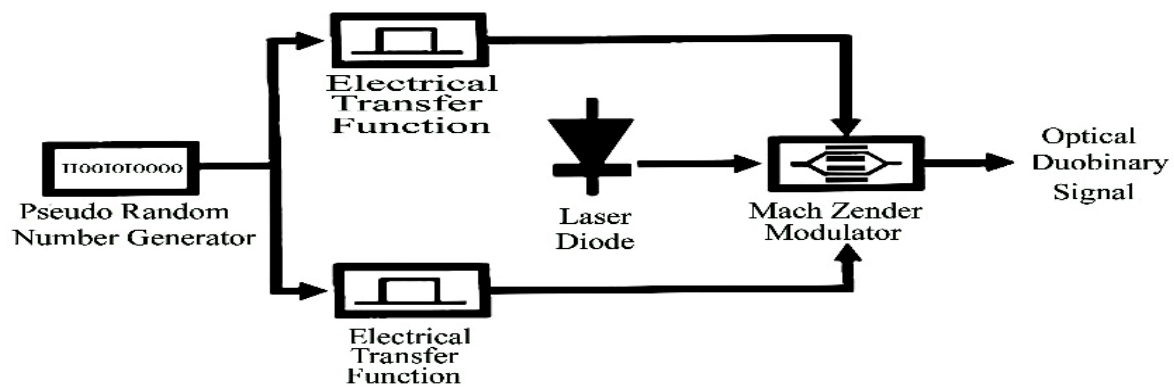

Figure 1.6 Mach-Zender Modulator (MZM)

Modulator splits an incoming light signal into two waveguide branches. The two branches experience different optical delays, depending on the voltage applied to each arm, and then recombine constructively or destructively. 


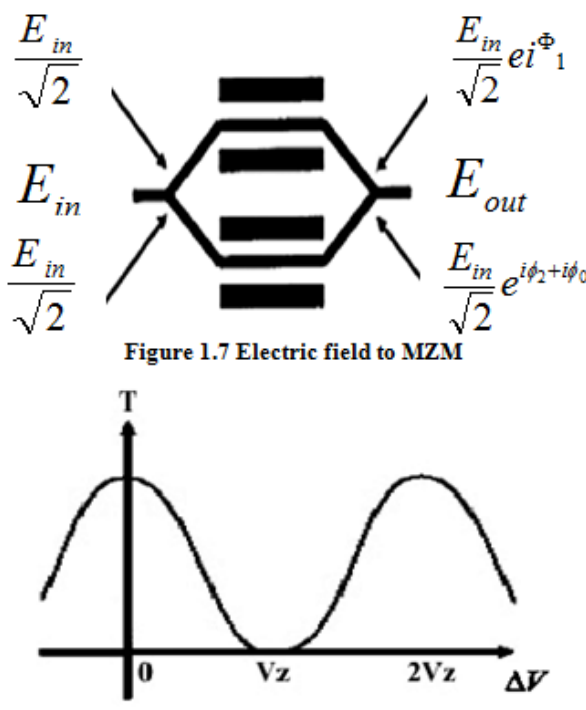

Figure 1.8 Electrodes voltage different to the MZM

If the electric field in the upper branch experiences a phase shift of

$\phi_{1}=\frac{v_{1}}{v_{\pi}} \pi(1.1)$

And the electric field in the lower arm experiences a phase shift of

$\phi_{2}=\frac{v_{2}}{\pi}$

The output electric field is given by [1],

$E_{\text {out }}=\frac{E_{\text {in }}}{\sqrt{2}}\left(e^{i \phi 1+} e^{i \phi 2+i \phi 0}\right)$ (1.3)

Where, $\Phi_{0}$ is the phase shift, the biasing voltage. Equation (1.3) in terms of voltage yields

$E_{\text {out }}=\sqrt{2} E_{\text {in }} e^{i\left(\frac{v_{1} v_{2}}{2 v \pi} \pi+\frac{\phi_{0}}{2}\right)} \cos \left(\frac{v_{1}-v_{2}}{2 v \pi} \pi-\frac{\phi_{0}}{2}\right)$ (1.4)

Using (1.4) and the definition $\Delta \mathrm{V}=\mathrm{V}_{1}-\mathrm{V}_{2}$, the output power of the $\mathrm{MZ}$ can be expressed

$\left|E_{\text {out }}\right|^{2}=2 E_{\text {in }}{ }^{2} \cos ^{2}\left(\frac{\Delta v}{2 v_{\pi}} \pi-\frac{\phi_{0}}{2}\right)$

And the chirp, which is the derivative of the phase of $E_{\text {out }}(\delta \omega=2 \pi \mathrm{d} \Phi(\mathrm{t}) / \mathrm{dt})$ is

$\delta \omega=\frac{\pi}{2 v_{p} i} \frac{d}{d t}\left(v_{1}+v_{2}\right)(1.6)$

The transmission through the MZ is sinusoidal with respect to the difference of voltages applied through the electrodes, $V_{1}$ and $V_{2}$. In addition, to have a chirp less output, equation (1.6) implies $V_{1}=-V_{2}$. This condition is the same as push-pull operation. The final step is to modulate the light with the three level duobinary signal, which implies a three-level optical duobinary signal [1].For an AM-PSK duobinary transmitter, the MZ modulator is biased so that when $\Delta V=0$ the transfer function is at a null. The delay-andadd element in figure 1.11 can be replaced by an analog low-pass filter (LPF) with a cutoff at $1 / 4$ the bit rate. This filter will also produce a three-level signal. The electrical LPF following the delay-and-add block represents the limited bandwidth of the driving electronics, inserted electrical filters, and electrical amplifiers. When the cutoff of this filter is chosen correctly, the spectral narrowing of the duobinary signal is actually beneficial. The problem with AM-PSK duobinary modulation is to generate a high-speed (>10Gbit/s) three-level electrical signal. Each MZ modulator is rate with the voltage, $V_{\pi}$, necessary to go from maximum to minimum transmission to the MZ. This Voltage is often higher than what can be generating with a bit error rate test set (BERT) and necessitates the use of electrical amplifiers. Amplification of a three-level signal is especially troublesome since amplifiers usually operate in saturation. This means that the middle-level, if not saturated already, will probably lie asymmetrically between the upper and lower levels. In order to achieve a good optical eye pattern, the electrical signal fed into the MZ modulator should have its middle-level located half-way between the high and low electrical levels. Often this is very difficult to achieve. A two-level scheme would be 
much simpler to implement for this reason. In addition, modulation between a "-1" and" +1 " requires a $2 V \pi$ voltage swingtwice as much as what is necessary for binary modulation.

Figure 1.9 Alternating phase duobinary
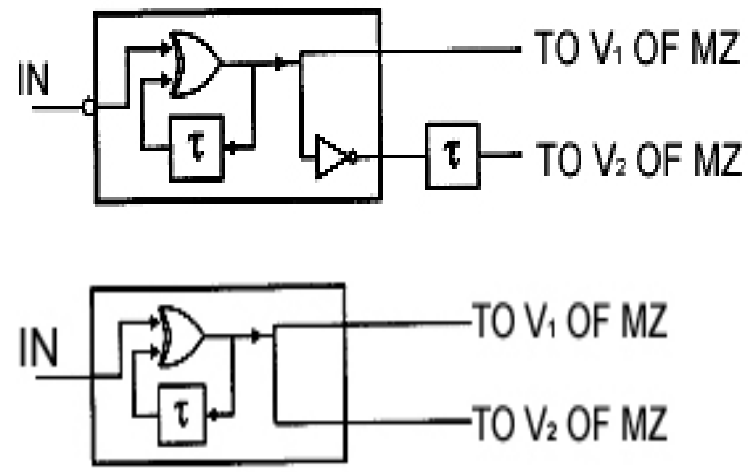

Figure 1.10 Blocked phase duobinary

There are two schemes to achieve a duobinary encoding with three-level electrical signals: alternating phase duobinary and blocked phase duobinary modulation (see figure 1.9 and 1.10). These schemes use a differential encoder and some delay elements. Unfortunately, both alternating-phase duobinary modulation and blocked phase duobinary modulation are not push-pull schemes, and hence the output from the MZ is chirped when operating in NRZ mode. On the other hand, the chirping is not seen when using an RZ pulse source since the chirping occurs at the transition edges of the electrical signals.

\subsubsection{Delay and Add Circuit and Duobinary Filter}

The binary data is two level representing 0 and 1.In the figure 1.5 the binary input data $A_{k}$ is precoded to $C_{k}$ but still it is a two level signal of having 0 and 1 . After delay-and-add operation, the result will be a three level signal $D_{k}$ with symbols of $(-1,0,1)$. Duobinary filter is an alternative method of producing encoded duobinary signal. Duobinary filter is a $5^{\text {th }}$ order Bessel filter called Thomson filter or may be electrical analog low pass filter [8]. A low-pass filter is a filter that passes low-frequency signal but attenuate (reduces the amplitude of) signals with frequencies higher than the cutoff frequency. It is sometimes called a high-cut filter, or treble cut filter when used in audio applications. A low-pass filter is the opposite of a high-pass filter, and a band-pass filter is a combination of a low-pass and a high-pass. In an electronic low-pass RC filter for voltage signals, high frequencies contained in the input signal are attenuated but the filter has little attenuation below its cutoff frequency which is determined by its RC time constant. Radio transmitters use low-pass filters to block harmonic emissions which might cause interference with other communications. One simple electrical circuit that will serve as a low-pass filter consists of a resistor in series with a load and a capacitor in parallel with the load. The capacitor exhibits reactance and blocks low-frequency signals, causing them to go through the load instead. At higher frequencies the reactance drops and the capacitor effectively functions as a short circuit. The combination of resistance and capacitance gives you the time constant of the filter $\tau=\mathrm{RC}$. The break frequency, also called the turnover frequency or cutoff frequency (in hertz), is determined by the time constant. One method of encoding technique is delay-and-add operation and another is duobinary filter. However the implementation based on the three level electrical signal generated by electrical analog low pass filter (duobinary filter) is the most cost effective and shows the best dispersion tolerance simultaneously. The bandwidth of an analog LPF will affect the pulse shape of the electrical signal and the driving voltage of an MZ modulator will also change the shapes of the optical duobinary signal because of the nonlinear electrical to optical transfer curve of an MZM. For achieving optimum performance, the bandwidth of analog low pass filter (LPF) should be set up based on the driving circuitry's and the modulator's bandwidth. So duobinary filter is the best approach for achieving encoded duobinary signal.

\section{Simulation Method}

Optsim is a simulation software for fiber optic communication system [12]. With the help of optsim software an optical fiber system can be designed with various components (such as various types of laser, fiber, receiver, optical amplifier, repeater, coupler, filter etc) and analyzed of signal at different points and models. For an optical fiber system the eye diagram, power spectrum, relative power, bandwidth, bit error rate, power level of a signal can be analyzed at any point by the software with scope. 
In figure 1.11, we designed a completed duobinary fiber optic system with a single-channel (150-nm) $90-\mathrm{km}$ OC-192 link to observe the performance of transmission and generation of duobinary data. There are two techniques to encode duobinary data in duobinary transmission system. Both systems are almost same but have a difference to the encoded portion in the transmitting side. The duobinary transmission is implemented with either a combination of (a) delay-and-add circuit or (b) a single electrical $5^{\text {th }}$ order Bessel low pass filter called duobinary filter. Frequency, Base band signal and wavelength spectrum measuring scopes are connected at the transmitting and receiving side. Also eye diagram measuring scopes are connected in both sides and systems in our simulation.

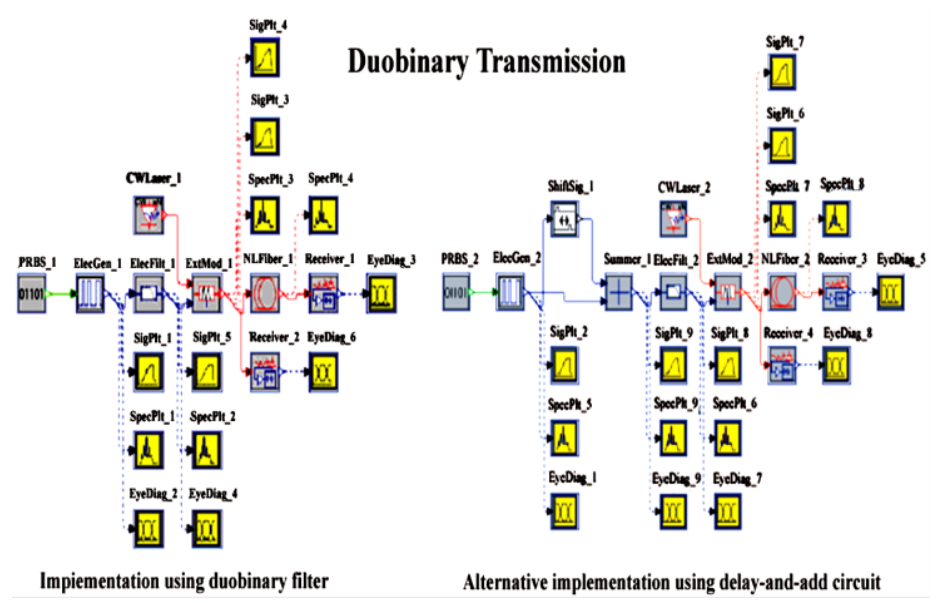

(a)

(b)

Figure 1.11 Simulated block diagram of (a) Duobinary filter (b) Delay -and -add circuit in duobinary transmission in fiber optic communication using Optsim software

\section{Results and discussion}

From these wave shapes it is defined that for encoding technique the duobinary filter is better than delay-and-add operation in duobinary transmission system. Eye diagram of duobinary encoding for both systems at the receiving side are shown in figure $1.12,1.13,1.14$ and 1.15 .

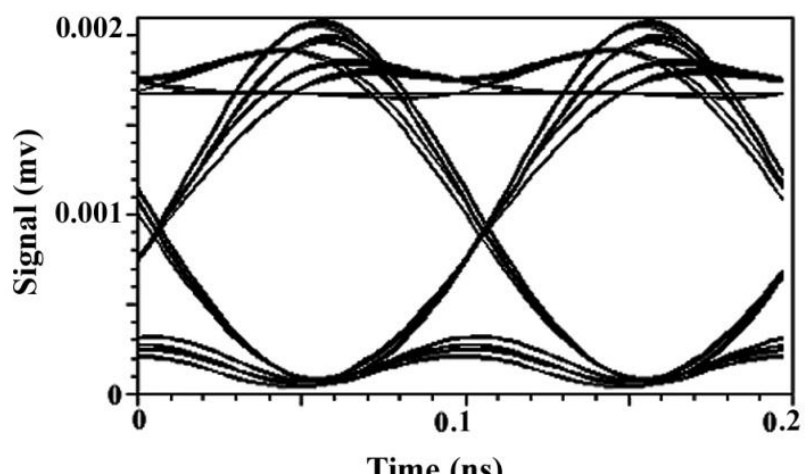

Time (ns)

Figure 1.12 Eye diagram of duobinary Filter at receiver-1 scope in duobinary transmission system

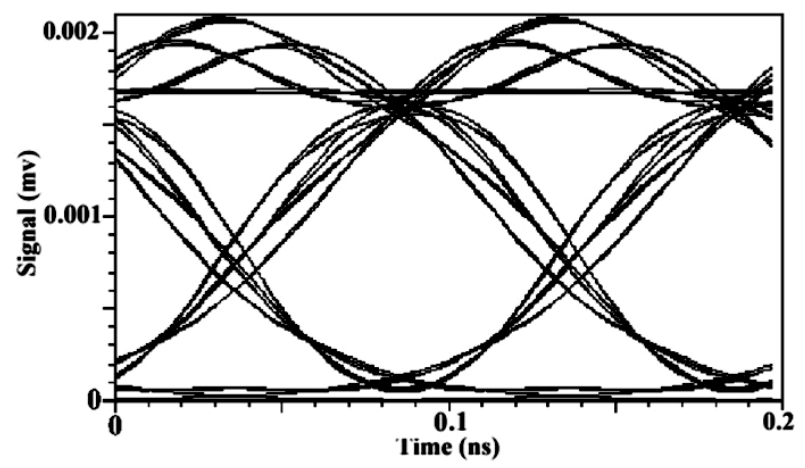

Figure 1.13 Eye diagram of delay-and-add operation at receiver-3 scope in duobinary transmission system 
But in the figure 1.12 and 1.13, the wave shapes of eye diagram are given from the Scopes which are connected at the receiver- 1 and receiver- 3 and eye diagram of figure 3.14 and 3.15 are connected at the receiver- 2 and receiver-4 of the system. From our simulation figure $1.12 \& 1.14$ represents eye diagram of duobinary filter and figure $1.13 \& 1.15$ are delay-and-add operation. Both of these two methods are used for encoding of duobinary data. But among these two methods duobinary filter is the best. Both the eye diagram of figure 1.12 $\& 1.13$ represent $2 \times 10^{-10}$ s time to horizontal axis and $2 \times 10^{-5}$ volt to vertical axis.

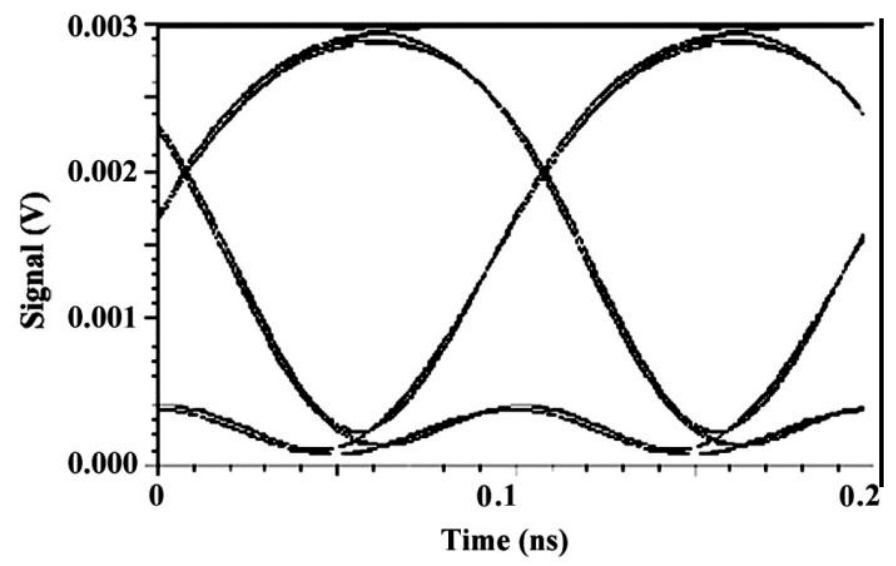

Figure 1.14 Eye diagram of duobinary filter at receiver-2 scope in duobinary transmission system

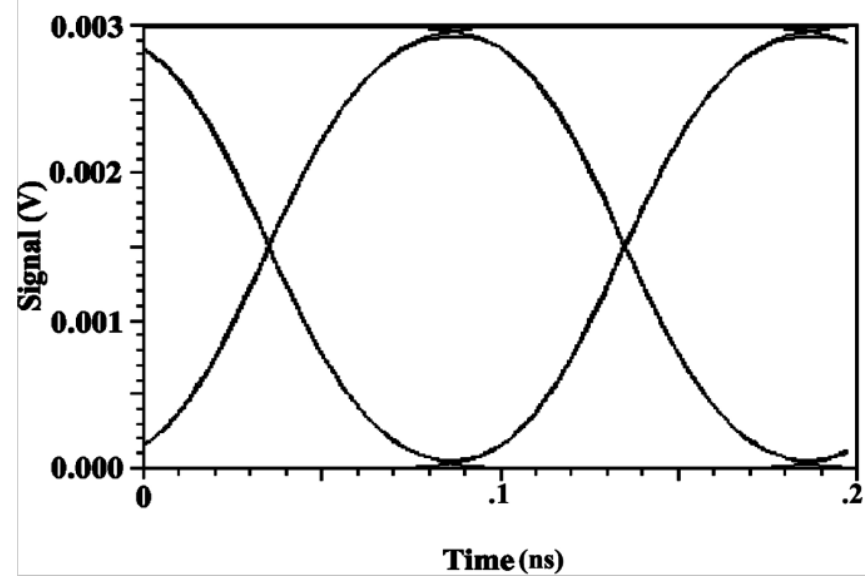

Figure 1.15 Eye diagram of delay-and-add Operation at receiver-2 scope in duobinary transmission system

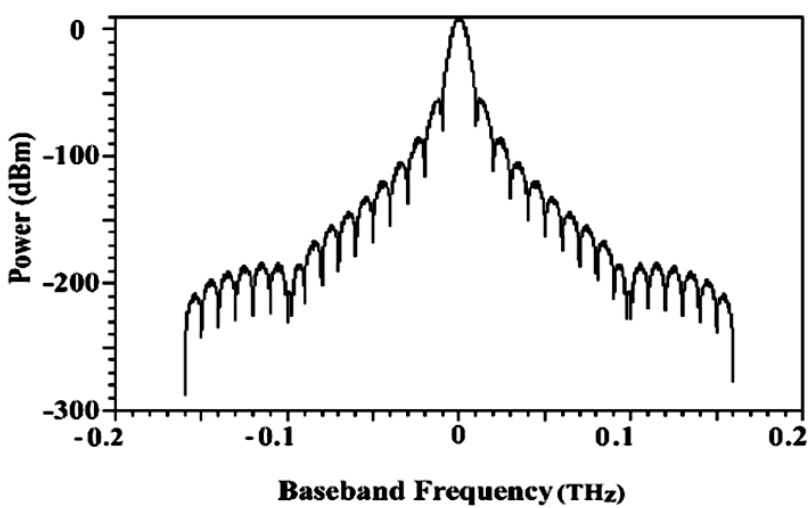

Figure 1.16 Frequency oscillation of base band signal of duobinary filter in duobinary transmission system 


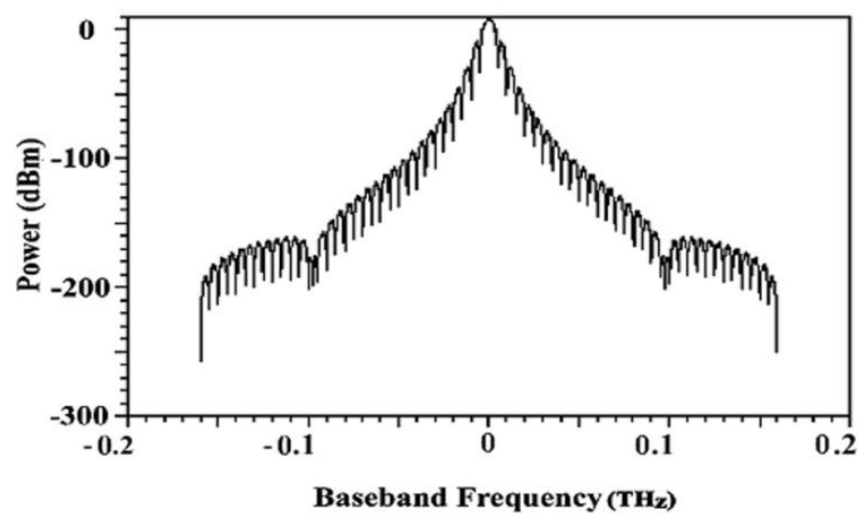

Figure 1.17 Frequency oscillation of base band signal of delay-and-add circuit in duobinary transmission system

Also figure $1.14 \& 1.15$ represent $2 \times 10^{-10} \mathrm{~s}$ time to horizontal axis and 0.003 volt to vertical axis. Comparing the eye diagram of figure 1.12 and 1.13 we can observe that the eye opening for the duobinary filter is higher than the delay-and-add operation. Also Peak distortion, noise margin and sensitivity of timing error of duobinary filter are higher than delay-and-add circuit. Again the same type of observation comes from figure $1.14 \& 1.15$ is that opening space of eye diagram of duobinary filter is big and better than delay-and-add operation. Frequency oscillation of encoded duobinary base band signal for both the systems are shown is figure $1.16 \& 1.17$ by the scope SpecPlt_2 which is connected to ElecFilt_2 (low pass filter) just before modulation. From these two figures it is observed that the oscillation of base band signal of delay-and-add circuit is higher than duobinary filter. So in this respect duobinary filter is better.

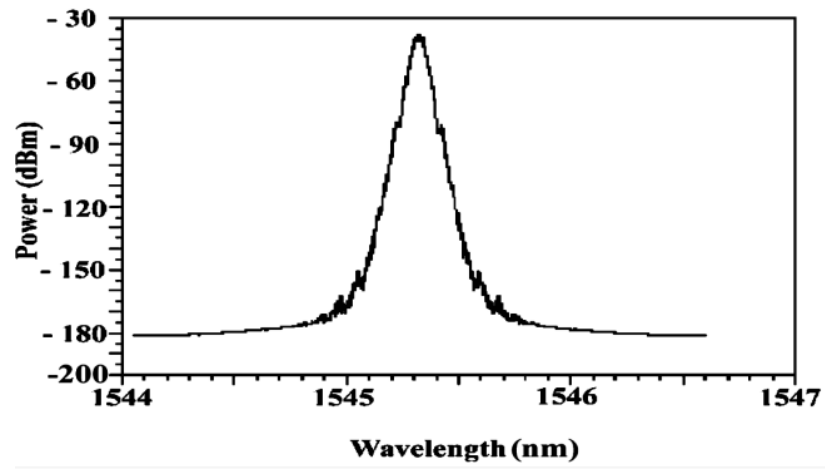

Figure 1.18 Wave length of duobinary filter at specplt_8 scope in duobinary transmission system

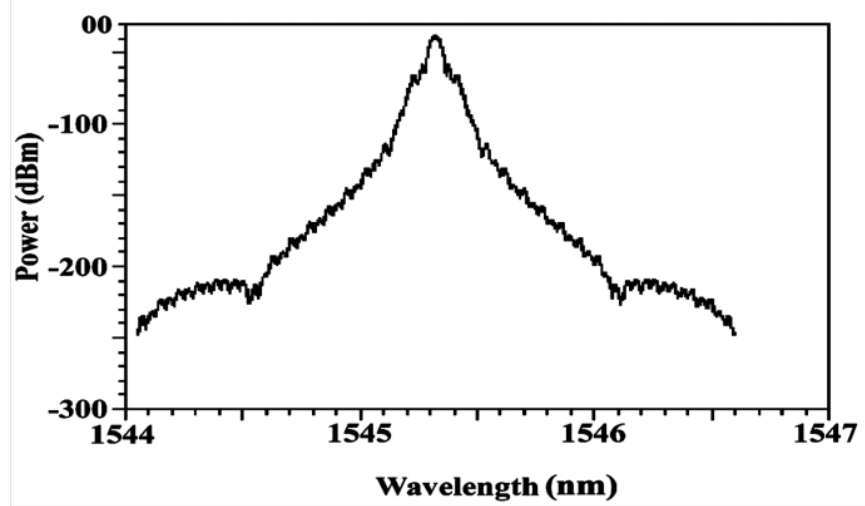

Figure 1.19 Wave length of delay-and-add operation at specplt_8 scope in duobinary transmission system

Figure1.18 \&1.19represent the wavelength of duobinary filter and delay-and-add operation of duobinary encoded system respectively. Wavelength is dependent on bandwidth. Frequency is inversely proportional to wavelength. In duobinary filter encoding technique from figure 3.18, we observed wave length of the optical duobinary signal is 1545.0 nanometer to 1545.6 nanometer (i.e. Bandwidth: 0.6 nanometer) on horizontal axis and output power is $-141.721 \mathrm{DBm}$. On the other hand from figure 3.19 the wave length of the 
delay-and-add circuit of optical duobinary signal is 1544.1 nanometer to 1546.6 nanometer (i.e. Bandwidth: 2.5 nanometer) on vertical axis and output power is $-285.293 \mathrm{DBm}$. So it is clear that the wave length and bandwidth of the duobinary filter is less and the output power is higher than delay-and-add circuit in duobinary transmission system

\section{Conclusion}

In this research Optsim software is used for simulation. The bandwidth, wavelength, noise margin and oscillation of the encoding technique are observed. Duobinary transmission can be done by either with duobinary filter or with delay-and-add circuit operation. Among these two technique duobinary filter having less wavelength, noise margin and oscillation than delay-and-add operation. For this reason the duobinary filter based duobinary transmission system has low cost for the fiber optic communication system due to reduction of the band width, wavelength and noise margin. So duobinary filter is better than delay-and-add circuit for duobinary transmission system in optical fiber communication system.

The optical fiber technology which is used in Bangladesh presently is phase system digital hierarchy (PHD). PHD has lass speed in data transmission compare to others. Now a days synchronous digital hierarchy (SDH) technology is used which has a speed of 64 STM. 1 synchronous transfer module $(\mathrm{STM})=155 \mathrm{Mbps}$. So calculated speed for $1 \mathrm{SDH}=(64.155) \mathrm{Mbps}=9.92 \mathrm{Gbps}$. Currently dense wavelength division multiplexing (DWDM) technique of fiber optic combination is installing in Bangladesh under Grameen phone mobile communication. DWDM has the speed of $80 \mathrm{SDH}=(80.9 .92) \mathrm{Gbps}=793.6 \mathrm{Gbps}$. So the researches can design the optimal bandwidth of an electrical low pass filter (Duobinary Filter) for the conventional Bangladeshi fiber 0ptic communication system (DWDM) with the speed 793.6 Gbps as future work.

\section{Acknowledgement}

It is my great pleasure to acknowledge the generous contribution of many individuals which have assisted me in writing this dissertation. At first I express my deep sense of gratitude and indebtedness to my supervisor, Mr. Russel Reza Mahmud, Assistant professor of department of Electrical \& Electronic Engineering, Ahsanullah University of Science \& Technology, for his kind help, advice, constant encouragement and valuable guidance throughout the course of this work. Mr. Russel Reza Mahmud has kindly reviewed this manuscript and provided me with many helpful suggestions.

I am expressing my deep appreciation and gratitude to all my respectable teachers, B.Sc. classmates and friends for their kind co-operation and constant encouragement throughout the research work.

Finally, I would like to thank my parents for their never ending support and inspiration during my research work.

\section{References}

[1] L. A. Jiang, "Propagation Properties of Duobinary Transmission in Optical Fibers," PhD thesis submitted to Massachusetts Institute of Technology, May, 1998.

[2] W. Kaiser, T. Wuth, M. Wichers and W. Rosenkranz, “A Simple System Upgrade from Binary to Duobinary,” National Fiber Optic Engineers Conference. pp. 1043- 1050, Germany, 2001.

[3] H. Kim and C. X. Yu, "Optical Duobinary Transmission System Featuring Improved Receiver Sensitivity and Reduced Optical Bandwidth," IEEE Photon. Technol. Lett., Vol. 14, No. 8, pp. 1205-1207, Aug, 2002.

[4] C. Xie, L. Moller and R. Ryf, "Improvement of Optical NRZ and RZ Duobinary Transmission Systems with Narrow Bandwidth Optical Filters,” IEEE Photon. Technol. Lett., Vol. 16, No. 9, pp. 2162-2164, Sep, 2004.

[5] P. Bravetti, L. Moller, G. Ghislotti, C. Cavalli, C. Gualandi and P. Bergamini, "Impact of Response Flatness on Duobinary Transmission Performance: an Optimized Transmitter with Improved Sensitivity," IEEE Photon. Technol. Lett., Vol. 16, No. 9, pp. 2159-2161, Sep, 2004.

[6] A. Rahman, M. Broman and M. Howieson, "Optimum Low Pass Filter Bandwidth for Generating Duobinary Signal for 40 Gb/S Systems,” Thin Film Technology Corp. Commerce Drive, North Mankato, MN, 56003, USA, 1980.

[7] Website: www.answers.com

[8] X. Gu, S.J. Dodds, L.C. Blank, D.M. Spirit, S.J. Pycock and A.D. Ellis, "Duobinary Technique for Dispersion Reduction in High Capacity Optical Systems- Modeling, Experiment and Field Trial,'IEEE Proc. Optoelectron, Vol.143, No. 4, August, 1996.

[9] J. Builting, "Introduction to Duobinary Encoding and Decoding," Elektor Electronics, pp. 50-52, January, 1990.

[10] Paul Williams, "Crossover Filter Shape Comparisons A White Paper from Linea Research Ltd," Linea Research ISS. B, July, 2006.

[11] B. Analui and A. Hajimiri, "Statistical Analysis of Integrated Passive Delay Lines," California Institute of Technology, Pasadena, CA 91125

[12] M. A. Barik, M. S. Hossain, M. A. G. Khan and S. M. A. Razzak, "Performance Analysis of Modulated Laser with Continuous Wave Laser in Fiber Optic Communication System,"CERIE 2010, Sylhet, Bangladesh, 11-13 January, 2010.

[13] R. Miller, "A Bessel Filter Crossover, and Its Relation to Other Types,"'The 105 Convention of the Audio Engineering Society, September 26-29, San Francisco, CA, 1998.

[14] Y. C. Lu, C. C. Wei and et al, " $2.5 \mathrm{~dB}$ the Sensitivity Improvement by Optimizing Driving Voltage of an MNZ and Electrical Filter Bandwidth of Optical Duobinary Transmission Systems," Optical Society of America, 2006.

[15] website: www.wikipedia.com

[16] Y. C. Lu, J. Chen, K. M. Feng and et al, "Improved SPM Tolerance and Cost- Effective Phase-Modulation Duobinary Transmission Over 230-Km Standard Single-Mode Fiber Using a Single Mach Zender Modulator,'IEEE Photonics Technology Letters, Vol.17, No.12, pp- 2754-2756, December, 2005. 
[17] J. C. Palais, "Fiber Optic Communication System," Pearson Education Fourth Edition, 2001.

[18] J. G. Proakis and M. Salehi, "Communication System Engineering," 2nd Edition, Singapore.

[19] M. S. Hossain, M. A.G. Khan, S. C. Majumder and M. I. Hossain, “Optimization of Fiber Optic Link Design,”Published in SUJSE. Vol.2, No.2. December, 2008.

[20] J. Zhou, N. Park, J. W. Dawson, K. J. Vahala, M. A. Newkirk and B. I. Miller, "Efficiency of Broadband Four-Wave Mixing Wavelength Conversion Using Semiconductor Traveling-Wave Amplifiers,'IEEE Photonics Technology Lett., Vol. 6, No. 1, January, 1994.

[21] O. Aso , M. Tadakuma and S. Namiki, "Four-Wave Mixing in Optical Fibers and Its Applications,"WP Team, Opto-Technology Lab., R \& D Div, Furukawa Review, Vol.5, No. 19, January, 2000.

[22] M.W. Maeda, W. B. Sessa, W. I. Way, A.Y. Yan, L. Curtis, R. Spicer and R.I. Laming, "The Effect of Four-Wave Mixing in Fibers on Optical Frequency-Division Multiplexed Systems," Journal of Light Wave Technology, Vol. 8, No. 9, pp. 22-26, September, 1990.

[23] P. E. Allen and D. R. Holberg, "C-MOS Analog Circuit Design,” Second edition, Oxford University Press, 2002.

[24] R. Fitzpatrick, "Electromagnetism and Optics," First edition, The University of Texas at Austin.

[25] K. Lizuka, "Engineering Optics," Third edition, Springer Series in Optical Sciences, 2007.

[26] P. E. Green, Jr, "Fiber to The Home," Wiley Survival Guides in Engineering and Science, 2006.

[27] K. Okamoto, "Fundamentals of Optical Waveguides," Second Edition, Okamoto Laboratory Ltd Ibaraki, Japan, 2006.

[28] T. Numai, "Fundamentals of Semiconductor Lasers," Springer Series in Optical Sciences, 2004

[29] R. Bansal, "Fundamentals of Engineering Electromagnetic," CRC Press, 2006.

[30] A. Ghatak, K. Thyagaragan, "Introduction to Fiber Optics," Cambridge University Press.

[31] R. W. Boyd, "Nonlinear Optics," Third Edition, New York press, 2007.

[32] E. Rosencher and B. Vinter, "Optoelectronics," Cambridge University Press, 2006

[33] F. Poly, A. Cucinotta and S Sellery, "Photonic Crystal Fibers," Springer Series in Optical Sciences, 2007.

[34] D. Bailey and E. Wright, "Practical Fiber Optics," First Edition, Newnes is an Imprint of Elsevier, 2003.

[35] B. J. Thompson, "Optical Science and Engineering," Taylor \& Francis Group, LLC, 2007.

[36] H. J. R. Dutton, "Understanding Optical Communications,” First Edition, International Technical Support Organization, 1998.

[37] L. Kazovsky, S. Bendetto and A. Willner, "Optical Fiber Communication System," Artech House, Inc. Boston.1996.

[38] A. Lender, "Correlative Digital Communication Techniques," IEEE Transaction on communication Technology, Vol. 12, pp.128135, Dec, 1964.

[39] K. O. Hill, D. C. Johnson et al, “CW Three Wave Mixing in Single Mode Optical Fibers,” Journal of Applied Physics, Vol. 49, No. 10, October, 1978

[40] S. D. Personic, "Receiver Design for Digital Fiber Optic Communication Systems," The Bell System Technical Journal, Vol.10, July 1973.

[41] A. J. Price and N. L. Mercier, "Reduced Bandwidth Optical Digital Intensity Modulation with Improved Chromatic Dispersion Tolerance," Electronics Letters, Vol.1, pp. 32-37, January 5, 1995

[42] S. W. Golomb, "Digital Communication With Space Application," Prentice Hall, Inc. Englewood Cliffs, NJ, 1964

[43] K.O.Hill, D. C. Johnson, B. S. Kawasaki and R. I. Macdonald, "CW Three Wave Mixing Insingle Mode Optical Fibers," J. Appl. Phys. Vol. 49, pp. 5098-5106, 1978

[44] N. Shibata, K. Nosu, K. Iwashita and Y. Azuma, "Transmission Limitation due to Four Wave Mixing in Single Mode Optical Fibers,” IEEE J. SELECT. Area Commun. Vol. 8, pp. 1068-1077, 1990.

[45] C. Lin, W. A. Reed, A. D. Pears0on and H. T. Shang, "Designing Single Mode Fibers for Near-IR ( $1.1 \mathrm{~mm}-1.7 \mathrm{~mm})$ Frequency Generation by Phase-Matched Four Wave Mixing in the Minimum Chromatic Dispersion Region,” Electron. Lett. Vol. 18, pp: 87$88,1982$.

[46] R.W. Tkach, A. R. Chraplyvy, F. Forghieri, A. H. Gnauck and R. M. Derosier, "Four Photon Mixing and High Speed WDM Systems," IEEE J. of Light Wave Tech. Vol. 13, pp. 841-849, 1995.

[47] F. Forghieri, R. W. Tkach, A. R. Chraplyvy and D. Marcuse, "Reduction Off Our Wave Mixing Crosstalk in WDM Systems Using Unequally Spaced Channels," IEEE Photonics Tech. Lett. Vol. 6, pp. 754-756, 1994.

[48] K. Okamoto, M. Ishii, Y. Hibino, Y. Ohmori and H. Toba, "Fabrication of Unequal Channel Spacing Arrayed Waveguide Grating Multiplexer Modules,” Electron. Lett. Vol. 31, pp. 1464-1465, 1995.

[49] M. M. Mano, "Digital Design," California State University, Los Angeles, Third Edition, 2004

[50] R. F. Coughlin, "Operational Amplifier Linear Integrated Circuits," Sixth Edition, 2007.

[51] R. A. Gaykwad, “Op-Amps and Linear Integrated Circuits,” Prentice Hall of India, Forth Edition, 2002. 